\title{
NGC 4261 and NGC 4697: Rejuvenated Elliptical Galaxies
}

\section{Citation}

Zezas, A., L. Hernquist, G. Fabbiano, and J. Miller. 2003. “NGC 4261 and NGC 4697: Rejuvenated Elliptical Galaxies." The Astrophysical Journal 599 (2): L73-77. https://doi.org/10.1086/380895.

\section{Permanent link}

http://nrs.harvard.edu/urn-3:HUL.InstRepos:41381785

\section{Terms of Use}

This article was downloaded from Harvard University's DASH repository, and is made available under the terms and conditions applicable to Other Posted Material, as set forth at http:// nrs.harvard.edu/urn-3:HUL.InstRepos:dash.current.terms-of-use\#LAA

\section{Share Your Story}

The Harvard community has made this article openly available.

Please share how this access benefits you. Submit a story.

\section{Accessibility}


Draft Version November 3, 2018

Preprint typeset using $\mathrm{LAT}_{\mathrm{E} X} \mathrm{X}$ style emulateapj v. 11/12/01

\title{
NGC 4261 AND NGC 4697: REJUVENATED ELLIPTICAL GALAXIES
}

\author{
A. Zezas, L. Hernquist, G. Fabbiano, J. Miller \\ Harvard-Smithsonian Center for Astrophysics, 60 Garden Street, Cambridge, MA 02138 \\ azezas@cfa.harvard.edu;lhernquist@cfa.harvard.edu; pepi@cfa.harvard.edu;jmmiller@cfa.harvard.edu
}

Draft version November 3, 2018

\begin{abstract}
Chandra images present evidence for a non-uniform spatial distribution of discrete X-ray sources in the elliptical galaxy NGC 4261. This non-uniform distribution is inconsistent with the optical morphology of NGC 4261 at greater than a $99.9 \%$ confidence level. Similar evidence is seen in one more elliptical galaxy (NGC 4697; 98\% confidence level) out of five cases we investigated. NGC 4261 and NGC 4697 have old stellar populations (9-15 Gyrs) and fine structure parameters of 1 and 0 respectively, suggesting no recent merging activity. On the basis of simulations of galaxy interactions, we propose that the Xray sources responsible for the non-uniform distribution are associated with young stellar populations, related to the rejuvenating fall-back of material in tidal tails onto a relaxed merger remnant, or shock induced star-formation along the tidal tails.
\end{abstract}

Subject headings: galaxies: elliptical and lenticular — galaxies: individual(NGC 4261; 3C270) — galaxies:interactions - X-rays: galaxies - X-ray: binaries

\section{INTRODUCTION}

Numerical simulations of galaxy interactions suggest that mergers of spiral galaxies can lead to the formation of elliptical galaxies (e.g. Toomre \& Toomre 1972; Barnes 1988, 1992; Hernquist 1992, 1993). These expectations are supported by optical imaging observations which show that several elliptical galaxies exhibit shells, ripples, arcs, counter-rotating cores, or faint tidal tails; structures which are interpreted as evidence for galaxy interactions or mergers (e.g. Schweizer et al. 1990).

The availability of high spatial resolution X-ray data allows us to investigate these indications using X-ray binary populations as probes of the star-formation histories of galaxies: High-Mass X-ray binaries (HMXBs) form much more efficiently than Low Mass X-ray binaries (LMXBs) (Kalogera \& Webbink, 1998; Portegies Zwart \& Verbunt, 1996), leading to a higher number of X-ray sources per star in young stellar populations. Thus, using number counts of discrete X-ray sources, we can identify regions of recent or enhanced star-formation within a galaxy. For example, the spatial distribution of LMXBs (which trace the old stellar populations) in early-type galaxies is expected to be smooth, generally following the distribution of optical star-light. On the other hand, if there are any sites of recent star-formation (e.g. triggered by galaxy interactions) they are expected to host HMXBs, the larger numbers of which (compared to the LMXBs) may result in an overall non-uniform X-ray source spatial distribution.

In this paper we present evidence for a non-uniform spatial distribution of X-ray sources in NGC 4697, an elliptical galaxy without any indication for merging activity from optical data, and we compare these results with similar observations of other early-type galaxies, finding a second example in NGC 4261. A more detailed investigation of the $\mathrm{X}$-ray source populations, their degree of non-uniformity and links to other merger activity diagnostics will be presented in a forthcoming paper.

\section{NGC 4261}

NGC 4261 is a nearby (35.1 Mpc for $\mathrm{H}_{\mathrm{o}}=75 \mathrm{~km} / \mathrm{s}$ ) E2 type galaxy, belonging to a poor group of galaxies (Garcia, 1993) but without any evidence for interaction with other members of the group. Optical observations show a $20^{\prime \prime}(\sim 3.4 \mathrm{pc})$ long dust lane along the NorthSouth axis of the galaxy (Martel et al. 2000 and references therein) and a spectacular nuclear dust disk $\left(\sim 2^{\prime \prime}\right.$; Jaffe et al. 1996, Ferrarese et al. 1996). Its optical isophotes in larger scales have a very strong boxy morphology (e.g. Peletier et al. 1990). Optical spectroscopy shows that its dominant stellar population has an age of $\sim 15 \mathrm{Gyr}$ (Trager et al. 2000). This galaxy also features two prominent radio jets (e.g. Birkinshaw \& Davies 1985, Jones et al. 2000) emanating from an active nucleus. The mass of the supermassive nuclear black-hole is estimated to be $(4.9 \pm 1.0) \times 10^{8} M_{\odot}($ Ferrarese et al. 1996) .

\subsection{Observations and Data Analysis}

NGC4261 was observed for $35 \mathrm{ks}$ (OBSID 834; PI M. Birkinshaw) with the ACIS-S3 CCD on-board the Chandra $\mathrm{X}$-ray Observatory. The spatial resolution of Chandra is $0.5^{\prime \prime}$ (van Speybroeck et al. 1997) corresponding to a physical resolution of $\sim 80 \mathrm{pc}$ at the distance on the galaxy. Although the observation was performed in $1 / 2$ subarray mode in order to mitigate pile-up on the nuclear source, the active field of view $\left(4.1^{\prime} \times 8.3^{\prime}\right)$ was large enough to cover the whole galaxy $\left(\mathrm{D}_{25}=4.07^{\prime}\right.$; de Vaucouleurs et al., 1991). The initial processing of the data and the results from the analysis of the nuclear spectrum are presented in Zezas et al. (2003). In this paper we concentrate on the spatial distribution of the discrete sources.

We used the wavdetect algorithm, within the CIAO v2.2 data analysis suite in order to search for discrete sources in four different energy bands: full band (0.3-7.0 keV), soft band $(0.3-2.0 \mathrm{keV})$ and hard band $(2.0-7.0 \mathrm{keV})$. The limit of $7.0 \mathrm{keV}$ was set in order to minimize contamination by the particle background, while the boundary of $2.0 \mathrm{keV}$ 
was chosen in order to limit the contribution of the diffuse emission (which is mainly thermal with $\mathrm{kT}=0.6 \mathrm{keV}$ ) only in the soft band. The source detection was performed in scales of $1,2,8$ and 16 pixels and a limiting probability of $\sim 1$ chance detection over this field. We found a total of 62 sources within the S3 CCD. Of those, 45 sources are within the D25 radius of NGC 4261, 40 of which have significance higher than $3 \sigma$ above the local background. The absorption corrected luminosities of the latter are above the Eddington limit for an $1.4 \mathrm{M}_{\odot}$ neutron star, ranging from $2.5 \times 10^{38} \mathrm{erg} \mathrm{s}^{-1}$ up to $4.4 \times 10^{39} \mathrm{erg} \mathrm{s}^{-1}$ (with two sources above $10^{39} \mathrm{erg} \mathrm{s}^{-1}$ ) assuming a power-law model with $\Gamma=1.7$ and Galactic line-of-sight $\mathrm{H}_{\mathrm{I}}$ column density $\left(5.8 \times 10^{20} \mathrm{~cm}^{-2} ;\right.$ Stark et al. 1992).

\subsection{Spatial distribution of X-ray sources}

Figure 1a shows an adaptively smoothed image of NGC 4261 in the full band, together with the D25 ellipse of the galaxy and the active field of the S3 ACIS-CCD. For reference in Figure 1b we present a DSS2 R-band image of the galaxy in the same scale, with the discrete sources marked by the best fit $3 \sigma$ ellipses to the spatial distribution of their photons ${ }^{1}$. From the X-ray image is clear that the spatial distribution of the discrete sources does not follow the distribution of optical star-light, but shows instead a distinct asymmetric pattern. In the following discussion we focus on the sources within the D25 ellipse.

In order to assess the probability that the non-uniform distribution may be observed by chance, we performed a Kolmogorov-Smirnov (KS) test to compare the distribution of the position angles (PA) of the sources (measured clockwise from the North-South direction), with (a) a uniform distribution and (b) the azimuthal profile of the optical light of the galaxy. For the latter we created a sample of "optical sources" based on the star-light distribution in the DSS2 R-band image, by randomly drawing position angles in $2^{\circ}$ sectors from a flat parent distribution (equivalent to assuming constant surface brightness within each $2^{\circ}$ sector). The number of draws in each sector was proportional to the fraction of the total galactic light included in it, after removing any strong point-like optical sources (most probably associated with foreground stars or background galaxies/AGNs). We found that both comparison distributions are inconsistent with the observed X-ray source azimuthal distribution above the $99.9 \%$ confidence level (Fig. 2a). This result holds even for model distributions with as few as 10 sources.

A search for irregularities in the azimuthal distribution of the PA of the sources, using the Bayesian blocks method (Scargle 1998; Scargle et al. 2003, in prep) also shows that there is an excess of sources between $\mathrm{PA}=140^{\circ}$ and $\mathrm{PA}=190^{\circ}$ at a confidence level greater than $99 \%$ (estimated from the prior of $\gamma=4.5$ used in the decomposition; however see Scargle et al. 2003, in $\operatorname{prep}^{2}$, for a caveat on this interpretation of the value of the prior). Therefore, we conclude that the spatial distribution of the X-ray sources is not the same as that of the optical light tracing the stellar population.

\section{OTHER EXAMPLES: NGC 4697}

\footnotetext{
${ }^{1}$ http://asc.harvard.edu/ciao/download/doc/detect_html_manual/

2 see also http://space.mit.edu/CXC/analysis/SITAR/functions.html
}

Indication for a different distribution of X-ray sources and optical star-light has been also seen in NGC 720 (Jeltema et al. 2003), which has relatively young stellar populations ( $\sim 5$ Gyrs; Trager et al. 2000). The X-ray sources in NGC 720 appear to trace out spiral arms, but performing the same analysis as above we confirm that this result is not statistically significant (Jeltema et al. 2003). Based on the KS test we find that the X-ray source distribution is different from the optical distribution or a constant at the $50 \%$ and $70 \%$ confidence levels respectively. Similarly, the Bayesian blocks method showed that any local enhancement of sources is significant only at the $63 \%$ confidence level.

We searched the Chandra archive for deep observations of nearby early-type galaxies, which also did not show any evidence for fine structure (Schweizer \& Seitzer 1992) or recent merging activity, in order to construct a comparison sample. We obtained data for NGC3379, NGC4636 and NGC4697, the general properties of which are presented in Table 1. We analyzed the data for these three galaxies in the same way as for NGC 4261 (§2.1). A KS test between the X-ray source distribution and the comparison distributions (calculated as described in \$2.2) showed that only in the case of NGC 4697 we can rule out at the $98 \%$ confidence level (Fig. $2 \mathrm{~b}$ ) that they belong to the same population $(\sim 95 \%$ probability that there is a local enhancement of sources based on Bayesian blocks). In the other two cases (NGC 3379 and NGC 4636) these confidence levels are $\sim 30 \%$ and $\sim 25 \%$ respectively, not allowing us to draw any definitive conclusions.

\section{DISCUSSION AND CONCLUSIONS}

Our results show evidence for a non-uniform spatial distribution of the X-ray source population in two nearby apparently normal elliptical galaxies. These galaxies have very low fine-structure parameters $(\Sigma=1$ for NGC 4261 and $\Sigma=0$ NGC4697) indicating that if they are merger products the merger event took place at least a few Gyrs ago. This is because merger simulations indicate that ripples, shells or strong tidal tails are not easily observable for much longer than $\sim 10^{9}$ yr after the relaxation of the merged system (e.g. Quinn 1984; Hernquist \& Quinn 1988, 1989). Moreover, faint traces of tidal tails surviving for much longer might not be easily detectable in optical observations because of their very low surface brightness (Mihos 1995). In fact, optical observations indicate stellar populations as old as 9 and 15 Gyrs in the nuclear regions of NGC 4697 and NGC 4261 respectively (Trager et al. 2000).

We propose that one or more localized star-formation events which occurred at most a few hundred Myr ago, are responsible for the discrepancy between the optical and X-ray morphology of these two galaxies. According to X-ray binary formation models, LMXBs are more susceptible to effects such as supernova kicks and common envelope phases (Kalogera \& Webbink, 1998) than HMXBs, leading to HMXB formation efficiencies 10 to 100 times higher than for LMXBs (Portegies-Zwart \& Verbunt 1996). In fig. 3 we plot the $\mathrm{L}_{\mathrm{X}} / \mathrm{L}_{\mathrm{B}}$ ratios of star- 
forming galaxies from the Einstein survey of Shapley, Fabbiano \& Eskridge (2002) together with the mean $\mathrm{L}_{\mathrm{X}} / \mathrm{L}_{\mathrm{B}}$ ratio for the discrete $\mathrm{X}$-ray sources in elliptical galaxies $\left(\log \left(\mathrm{L}_{\mathrm{X}} / \mathrm{L}_{\mathrm{B}}\right)=-3.05 \pm 0.4^{3} ;\right.$ Athey 2003$)$. From this plot is clear that even after accounting for a $50 \%$ contribution from the diffuse emission in star-forming galaxies (e.g. Zezas et al. 2001), the latter have systematically higher $\mathrm{L}_{\mathrm{X}} / \mathrm{L}_{\mathrm{B}}$ ratios than the X-ray binary component of early type galaxies. This together with the fact that individual HMXBs have significantly lower X-ray to B-band flux ratios than LMXBs (van Paradijs \& McClintock 1995) supports the picture that HMXBs are forming much more efficiently than LMXBs.

Recently Barnes (2003) suggested that shock-induced star-formation can explain the star-forming activity observed along the tidal tails of "The Mice" (Stockton 1974; de Grijs et al. 2003). According to this model shock waves developing along the tidal tails can compress the neutral gas and trigger star-formation. This picture is consistent with the tail-like distribution of the observed X-ray sources in NGC 4261, if the tidal tails are projected against the body of the merger remnant. If the age of this young stellar population is less than a few hundred Myr, it forms X-ray binaries much more efficiently than the populations in the relaxed merger remnant. On the other hand its optical emission is diluted by the optical light of the much stronger old population making its detection in the optical band very difficult. Depending on the strength of the star-formation, this may result in an overall projected spatial distribution of X-ray sources which is inconsistent with that of the star-light. This star-formation event can be well-approximated by an instantaneous burst, in which case the young X-ray source populations are expected to decay in a few hundred Myr after the passage of the shock. Therefore, we estimate that the shock should propagate with a speed of $\sim 100 \mathrm{~km} \mathrm{~s}^{-1}$ in order to cover the length of the region we observe X-ray sources $(20.5 \mathrm{kpc})$, within the timescale of HMXB formation ( $\sim 200 \mathrm{Myr})$. This speed is realistic for shock waves developing in the interstellar medium.

Alternatively, N-body simulations show that structures resembling dwarf galaxies can form in the tidal tails as a merger completes (Barnes \& Hernquist 1992, 1996). These structures remain bound to the body of the remnant, orbiting it. Because the objects formed in this manner have a range of binding energies in the potential well of the remnant, the characteristic time for them to fall back onto the remnant can be much longer than the time for the body of the remnant to relax (Hernquist \& Spergel 1992; Mihos \& Hernquist 1996). Thus, depending on the orbital distribution, the remnant will eventually lose evidence for a merger, while tidal dwarfs continue to orbit at large radii. The interaction between the body of the merger and the condensations in the tidal tails can trigger small scale star-formation events and locally enrich the galaxy with a young stellar population.

As was mentioned earlier, a young stellar population can form X-ray binaries very efficiently, while it is very difficult to detect it against the much brighter stellar populations of the merger remnant. Therefore, depending on the strength of the star-formation, this may result in an overall spatial distribution of X-ray sources which is inconsistent with that of the star-light. Since fall-back is estimated to occur for several Gyrs after relaxation, we expect that even elliptical galaxies with stellar populations of $\sim 10$ Gyrs may exhibit non-uniform X-ray source distribution, if they are the end-points of galaxy mergers.

Although the spatial distribution of the sources in NGC 4261 indicates that they are associated with the tidal tails, probably both mechanisms can produce populations of numerous, young X-ray binaries in merger remnants. Given the timescales of the orbits of dwarf galaxies (up to $1 \mathrm{Gyr}$ ) or the velocities of the shocks along the tidal tails, and the lifetime of HMXBs (up to $\sim 100 \mathrm{Myr}$ ), we would expect long periods during which these galaxies have uniform X-ray source distributions. These periods are expected to be longer in the more evolved systems since the dwarf companions with short orbits are accreted first, which is consistent with our finding that this phenomenon appears in only a few of the most evolved galaxies examined. Studies of a more complete sample of elliptical galaxies and merging galaxies in their latest stages of merging will allow us to further investigate this hypothesis.

We thank the referee (J. Rose) for a very helpful report. We also thank J. Barnes for providing results prior to publication, and E. O'Sullivan and A. Athey for useful discussions on the X-ray emission of X-ray binaries in elliptical galaxies. We thank P. Ratzlaff and J. J. Drake for making available their code for Bayesian blocks analysis. This work has been partly supported by NASA Grants G01-2116X, G01-3150X, and ATP NAG5-12140 and NSF grants ACI 96-19019, AST 98-02568, AST 99-00877, and AST 00-71019.

\section{REFERENCES}

Athey, A. E. 2003, PhD Thesis, University of Michigan

Barnes, J. E. 1988, ApJ, 331, 699

Barnes, J. E. 1992, ApJ, 393, 484

Barnes, J. E. 2003, MNRAS, submit.

Barnes, J. E. \& Hernquist, L. 1996, ApJ, 471, 115

Barnes, J. E. \& Hernquist, L. 1992, Nature, 360, 715

Birkinshaw, M. \& Davies, R. L. 1985, ApJ, 291, 32

Colbert, J. W., Mulchaey, J. S., \& Zabludoff, A. I. 2001, AJ, 121, 808

Ferrarese, L., Ford, H. C., \& Jaffe, W. 1996, ApJ, 470, 444

Garcia, A. M. 1993, A\&AS, 100, 47

de Grijs, R., Lee, J. T., Clemencia Mora Herrera, M., Fritze-

v. Alvensleben, U., \& Anders, P. 2003, New Astronomy, 8, 155

Hernquist, L. 1992, ApJ, 400, 460
Hernquist, L. 1993, ApJ, 409, 548

Hernquist, L. \& Quinn, P. J. 1988, ApJ, 331, 682

Hernquist, L. \& Quinn, P. J. 1989, ApJ, 342, 1

Hernquist, L. \& Spergel, D. N. 1992, ApJ, 399, L117

Jaffe, W., Ford, H., Ferrarese, L., van den Bosch, F., \& O'Connell, R. W. 1996, ApJ, 460, 214

Jeltema, T. E., Canizares, C. R., Buote, D. A., \& Garmire, G. P. 2003, ApJ, 585, 756

Jones, C., Forman, W., Vikhlinin, A., Markevitch, M., David, L., Warmflash, A., Murray, S., \& Nulsen, P. E. J. 2002, ApJ, 567, L115

Kalogera, V. \& Webbink, R. F. 1998, ApJ, 493, 351

Martel, A. R., Turner, N. J., Sparks, W. B., \& Baum, S. A. 2000, ApJS, 130, 267

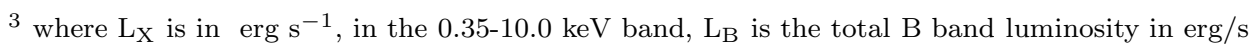


Mihos, J.C. 1995, ApJ, 438, L75

Mihos, J.C. \& Hernquist, L. 1996, ApJ, 464, 641

van Paradijs, J. \& McClintock, J. E. 1995, X-ray Binaries, eds. W.H.G. Lewin, J. van Paradijs, and E.P.J. van den Heuvel (Cambridge: Cambridge Univ. Press), p. 58, 58

Peletier, R. F., Davies, R. L., Illingworth, G. D., Davis, L. E., \& Cawson, M. 1990, AJ, 100, 1091

Portegies Zwart, S. F. \& Verbunt, F. 1996, A\&A, 309, 179

Quinn, P. J. 1984, ApJ, 279, 596

Scargle, J. D. 1998, ApJ, 504, 405

Schweizer, F. 1998, Saas-Fee Advanced Course 26: Galaxies: Interactions and Induced Star Formation, 105

Schweizer, F. \& Seitzer, P. 1992, AJ, 104, 1039

Schweizer, F., Seitzer, P., Faber, S. M., Burstein, D., Dalle Ore, C. M., \& Gonzalez, J. J. 1990, ApJ, 364, L33

Shapley, A., Fabbiano, G., \& Eskridge, P. B. 2001, ApJS, 137, 139 van Speybroeck, L. P., Jerius, D., Edgar, R. J., Gaetz, T. J., Zhao, P., \& Reid, P. B. 1997, Proc. SPIE, 3113, 89

Stark, A. A., et al. 1992, ApJS, 79, 77

Stockton, A. 1974, ApJ, 187, 219

Toomre, A. \& Toomre, J. 1972, ApJ, 178, 623

Trager, S. C., Faber, S. M., Worthey, G., \& González, J. J. 2000, AJ, 119,1645

de Vaucouleurs, G., de Vaucouleurs, A., Corwin, H. G., Buta, R. J., Paturel, G., \& Fouque, P. 1991, Springer-Verlag Berlin Heidelberg New York

Zezas A., Birkinshaw M., Worrall D., Peters A., \& Fabbiano, 2003, ApJ, submitted

Zezas, A., Fabbiano, G., Prestwich, A., Murray, S., \& Ward, M. 2001, ASP Conf. Ser. 249: The Central Kiloparsec of Starbursts and AGN: The La Palma Connection, 425 
TABLE 1

Properties of the Galaxies

\begin{tabular}{lcccc}
\hline \hline \multicolumn{1}{c}{ Galaxy } & Hubble Type & Distance $^{\dagger}(\mathrm{Mpc})$ & $\Sigma^{\ddagger}$ & Notes \\
\hline NGC 4261 & E2 & 32.5 & 1.0 & dust, quadrupole structure (a) \\
& & & & age $\sim$ 15 Gyrs (b) \\
NGC 3379 & E1 & 8.1 & 0.0 & in group, age 5 Gyrs (b) \\
NGC 720 & E5 & 28.0 & - & complex X-ray morphology (c) \\
NGC 4636 & E0 & 15.0 & - & age $\sim 9$ Gyrs (b) \\
NGC 4697 & E6 & 15.9 & 0.0 & \\
\hline
\end{tabular}

${ }^{\dagger}$ Galaxy distance assuming $\mathrm{H}_{\mathrm{O}}=75 \mathrm{~km} / \mathrm{s} / \mathrm{Mpc}$.

${ }^{\ddagger}$ Fine structure parameter from Schweizer \& Seitzer, 1992.

References: (a) Colbert, Mulchaey \& Zabludoff, 2001; (b) Trager et al. 2000; (c) Jones et al. 2002

FIG. 1. - Left: (a) A full band (0.3-7.0 keV) adaptively smoothed Chandra image of NGC 4261; the D25 ellipse is shown by the white ellipse. The white box indicates the active field of the ACIS-S3 chip. The green ellipses indicate the position of the sources in the central, saturated, region of the image. Right: (b) The R-band DSS2 image of the galaxy with the X-ray sources marked by the red ellipses. The D25 ellipse is again shown by the white ellipse.
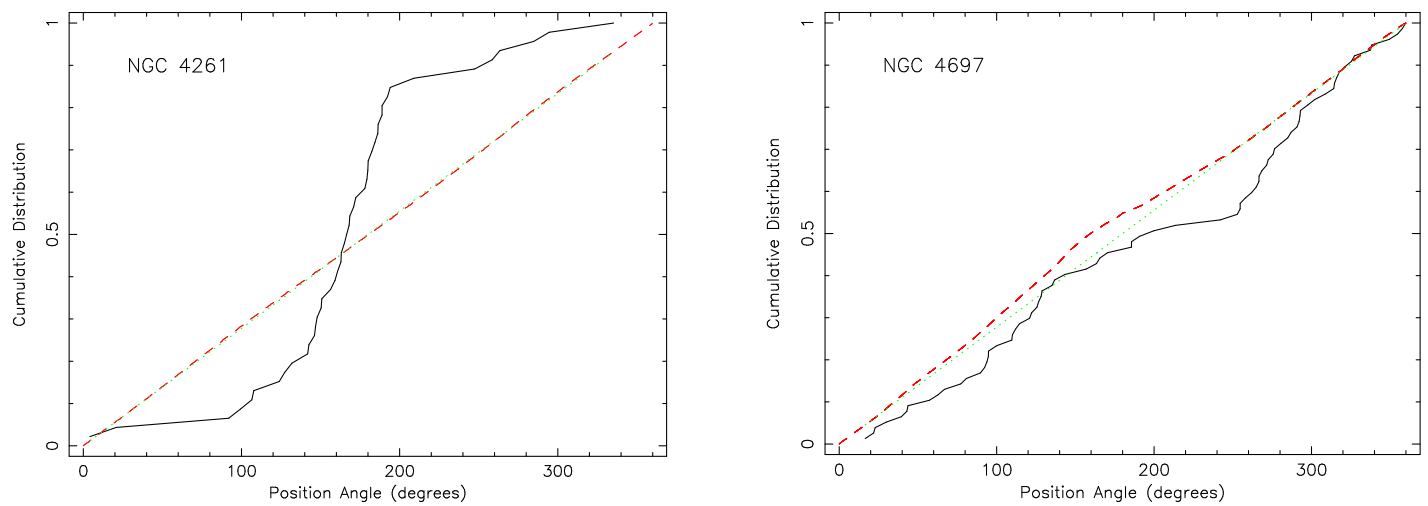

FIG. 2.- (a) Comparison between the cumulative distribution of the position angles of the X-ray sources within the D25 ellipse of NGC 4261 with the azimuthal distribution of its optical light (dashed red line) and a constant (dotted green line). (b) The same plot but for NGC 4697.

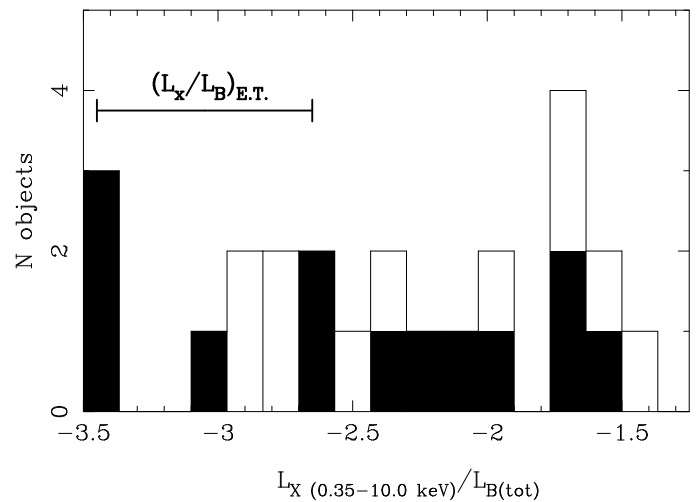

FIG. 3.- Histogram of the X-ray (0.35-10.- keV) to total B-band luminosity ratio of spiral galaxies later than Sc type (T $>6)$ from the Einstein sample (Shapley et al. 2001). The shaded regions show only the X-ray detections whereas the unshaded regions show both the detections and non-detections (upper limits). The horizontal line gives the range of $\mathrm{L}_{\mathrm{X}} / \mathrm{L}_{\mathrm{B}}$ for the discrete source populations of elliptical galaxies (Athey 2003). 
This figure "f1.jpg" is available in "jpg" format from: http://arxiv.org/ps/astro-ph/0310567v1 
This figure "f2.jpg" is available in "jpg" format from: http://arxiv.org/ps/astro-ph/0310567v1 